\title{
Estrategias de inclusión en contextos escolares*
}

\section{Inclusion strategies in school contexts}

Melba Ximena Figueroa Ángel ${ }^{* *}$

ORCID: 0000-0003-0420-1590

Universidad Santo Tomás, Colombia

\section{Carolina Gutiérrez de Piñeres Botero}

ORCID: 0000-0001-9079-3670

Universidad Santo Tomás, Colombia

Jeyson Velázquez León

Universidad Santo Tomás, Colombia

Recibido: 16 de mayo de 2016

Revisado: 13 de julio de 2016 Aceptado: 3 de septiembre de 2016

\section{Resumen}

En este artículo se presenta una revisión de las estrategias de inclusión social en la escuela presentadas en artículos científicos y textos, las cuáles fueron identificadas a través del análisis de contenido de tipo categorial en cuatro categorías; competencia, singularidad, vínculos y participación. Se encuentran aportes desde los avances en la comprensión de la inclusión a partir de estrategias que trascienden su nexo con la diversidad en su concepción como minoría, y la necesaria conexión entre el reconocimiento de la singularidad para la construcción de vínculos, el desarrollo de competencias y su incidencia en la participación como elemento clave de la inclusión.

Palabras clave autor: inclusión, competencia, vínculo, singularidad, participación, estrategias, escuela.

\section{Abstract}

This article presents a review of the social inclusion strategies at schools mentioned in scientific articles

Artículo de investigación. Producto de la investigación "Aplicación y evaluación de estrategias participativas para la movilización de significados y acciones en torno a la inclusión social de niños, niñas y adolescentes en contextos familiares y escolares”, grupo de investigación: Psicología, ciclo vital y derechos, número 12. (doi: http://dx.doi.org/10.15332/s1794-9998.2017.0001.01)

* Melba Ximena Figueroa Ángel. Universidad Santo Tomás. Dirección postal: Campus San Alberto Magno, Autopista Norte calle 205, vía Arrayanes km 1, Bogotá, Colombia. Correo electrónico: melbafigueroa@usantotomas.edu.co 
and books. These strategies were identified through categorical content analysis in four categories: competency, singularity, bonding and participation. There are contributions from strategies that go beyond their connection to diversity from its idea of "minority", and the necessary connection between the recognition of the uniqueness for link building, skill development and their impact on participation as a key element of inclusion.

Keywords: inclusion, competency, bonding, singularity, participation, strategies, school.

\section{Introducción}

En la actualidad se encuentra un panorama social, político, económico y educativo marcado por un acento en la inclusión social; políticas, comprensiones académicas, iniciativas públicas y privadas emergen como alternativa en la lucha contra la exclusión, tema que ha cobrado importancia en los últimos 30 años a partir de las reflexiones de la economía acerca de la relación exclusión-desarrollo (Sen, 2000).

Por lo tanto, el interés por la inclusión ha sido recogido por diferentes disciplinas, en tanto que este concepto implica aristas que complejizan las acciones al respecto. Así, se presentan comprensiones que van desde las posturas asociadas al progreso económico hasta la discusión sobre integración versus inclusión (UNESCO, 2005) en un escenario clave para el desarrollo social e individual: la escuela, la cual "debe ser democrática, científica e investigadora, el centro de la acción educativa es el alumno y su desarrollo como sujeto en el proceso, que garantiza el éxito de todos, es reflexiva, crítica, abierta y enraizada en el medio" (Rogero, 2012, p. 57).

Así, en la escuela, se gestan procesos de inclusión que inician con la atención a la discapacidad y posteriormente se amplía a la diversidad con su consecuente discusión sobre su viabilidad y coherencia social (Batallán y Campanini, 2007), y por lo tanto, se convierte en fuente de experiencias que se requiere sistematizar, en este caso, como estrategias que puedan favorecer dichos procesos.

De esta manera, se realiza una revisión documental con el objetivo de describir diferentes estrategias de inclusión social en la escuela para situaciones de vulnerabilidad de niños y adolescentes, a partir de la revisión de artículos de investigación y textos, sistematizados a través del análisis de contenido de tipo categorial, en cuatro categorías: competencias, singularidad, vínculos y participación.

\section{Referentes conceptuales}

\section{Inclusión social}

De acuerdo con Oakley (1995), la inclusión surge necesariamente como oposición a la exclusión social, término que se acuña en Francia en 1974 para referirse a personas con discapacidad mental o física, padres solteros, toxicómanos, entre otros individuos sin seguridad social, extendiéndose en la década de 1980 a los grupos con desventajas sociales, por lo cual se ve estrechamente relacionada con las rápidas trasformaciones económicas y sus consecuentes modificaciones en las relaciones sociales; el aumento del desempleo, las nuevas estructuras familiares, la disminución de la solidaridad y la ruptura de los vínculos sociales, simbólicos, económicos e institucionales que permitían a los sujetos percibirse como miembros de la sociedad (Oakley, 1995).

En ese sentido, la inclusión:

Se refiere a las oportunidades: representa la suma de los factores necesarios para que un individuo disfrute de una vida segura y productiva como un miembro totalmente integrado en la sociedad, independientemente de su raza, origen étnico, género u orientación sexual (Americas Quarterly, 2013, p. 3). 
De lo anterior emerge la reflexión sobre lo que significa ser miembro de una sociedad, que implica la percepción de pertenencia y respeto por la singularidad. De ahí que la inclusión se refiera al grado en que los individuos experimentan la satisfacción de sus necesidades enmarcadas en estas dos categorías (Shore, Randel, Chung, Dean, Holcombe, Singh, 2011).

De igual manera, se hace énfasis en el papel de la sociedad como agente de la inclusión, ya que es el grupo el que favorece los dispositivos para que la pertenencia y autenticidad se consoliden en cada individuo (Jansen, Otten, Van Der Zee, Lise, 2014).

Al respecto, se encuentran construcciones sociales en términos de políticas que acompañan los procesos de inclusión. Aun así, se revela un escenario social en el que situaciones sociales de vulnerabilidad aquejan a los miembros de la sociedad, especialmente a determinados sectores de la infancia (Chana, 2007) y en relación con la educación específicamente, ya que en América Latina se presentan desigualdades en el acceso que afectan a niños y a niñas de medios económicos desfavorecidos (Blanco, 2006).

En este trabajo, a partir de la revisión de las comprensiones de la inclusión, se retoma una definición de inclusión que da cuenta de las cuatro categorías que lo orientan:

La inclusión es una forma de interacción en la que los interlocutores se reconocen mutuamente como sujetos sociales competentes, singulares y dignos de reconocimiento, todo lo cual promueve la activa participación de cada uno y la formación de vínculos afectivos (Bernal, Figueroa, Gutiérrez de Piñeres y Jaramillo, 2011).

\section{Participación}

La participación aparece como categoría relevante para la gestión de la inclusión, y dado que la escuela es la institución que históricamente se ha considerado como una fuente de oportunidades para el desarrollo de las personas, es ella la que recrea la tensión entre la integración, entendida como el ajuste de los sujetos a las instituciones (Figueroa, 2010), y la inclusión definida por González (2008) como un propósito que abarca a todos los alumnos, conllevando a no excluir a nadie de la formación a la que tiene derecho por razones de justicia y democracia. Por otro lado, Moriña y Parrilla (2006) describen que la educación inclusiva se refiere a la escuela y al salón de clases como una comunidad en la que se debe promover y respetar los derechos de todos los alumnos ante su aprendizaje junto al de sus pares enmarcado desde el currículo común.

Por lo tanto, la inclusión se entendería como un proceso que fomenta la participación y pertenencia de todo el alumnado, y junto con ello, la superación de los obstáculos que conllevan a procesos de exclusión (Booth, Ainscow, Black-Hawkins, Vaughan y Snow, 2000). Teniendo en cuenta que dicha participación implica tres dimensiones: el ser parte, que se refiere a la pertenencia de los sujetos; el tener parte, como la conciencia de los deberes, derechos, ganancias y pérdidas; y el tomar parte, como el logro de la realización de acciones (Oraisón y Pérez, 2006).

\section{Singularidad}

Esta categoría se deriva de la tensión entre lo colectivo y lo singular; es decir, la pertenencia a un grupo respetando la diferencia. Al respecto Corcuff (2009) en una reflexión sobre la propuesta de Bourdieu (1980) sobre este desafío plantea:

Pensar lo colectivo y lo singular, lo colectivo dentro de lo singular, a través de un verdadero singular colectivo entendido como un ensamblaje singular de piezas colectivas... Solo estaríamos hechos de experiencias colectivas, pero la suma de esas experiencias y su orden nos harían completamente singulares, únicos (p. 14).

El concepto de singularidad en los procesos de inclusión, implica su reconocimiento en los estudiantes, lo cual se ha desarrollado en las escuelas durante las últimas décadas. Para Blanco (2008), la inclusión "exige la adaptación de la enseñanza a la diversidad de necesidades educativas del 
alumnado, que son fruto de su procedencia social y cultural y de sus características individuales en cuanto a motivaciones, capacidades e intereses" (p. 41). Es decir, la singularidad es fruto de las interacciones y los contextos en los cuales se encuentra inmerso el estudiante, y son estas diferencias las que se deben convertir en una prioridad para el abordaje de la inclusión.

\section{Competencia}

La escuela, en su función de educar en saberes y habilidades para el desarrollo de una sociedad, se ha caracterizado históricamente por requerir estudiantes con características cognitivas, culturales y económicas similares para cumplir con este propósito, y dado que la inclusión presenta el reto de la singularidad, es en la cuestión sobre las competencias en donde se puede encontrar un camino, dado que estas aparecen en el panorama educativo como alternativa a las comprensiones sobre la inteligencia y el umbral de educabilidad de acuerdo con su medición.

Así, se presenta la posibilidad de postular el comportamiento inteligente que se demuestra ante unos requerimientos determinados de acuerdo con el qué y el cómo (Varela y Ribes, 2002), liberándose del imperativo de lo biológico y la homogeneidad cultural y económica como menester para la participación en escenarios escolares y productivos.

De ahí que la comprensión de las competencias como "estructuras mentales internas, en el sentido de que son aptitudes, capacidades o disposiciones inherentes al individuo" (OCDE, 2005, p. 8) requiere dar el salto a su comprensión en relación con el contexto, dado que si se pretende la inclusión de los individuos en la sociedad, se requiere atribuir a la competencia la potencialidad de desarrollar aprendizajes significativos, duraderos y funcionales, que le permitan a sus miembros actuar sobre el mundo (Martín y Solari, 2011). De esta manera, las competencias favorecerían una escuela "de mayor calidad, una escuela que permita que todos aprendamos, y que lo hagamos con un concepto del aprendizaje bastante más intere- sante que el de declarar informaciones verbales, tal y como en este momento puede estar pasando" (Martín y Solari, 2011, p. 20).

\section{Vínculos}

Según Gairín e Iglesias (2008), los vínculos son un importante motor de las interacciones, que en últimas, se convierten en el soporte de la inclusión si se piensa cómo desde el aula de clase se construyen relaciones atravesadas por funciones como la compañía social, el apoyo emocional, la guía cognitiva y consejos, la regulación social, la ayuda material y de servicios, el acceso a nuevos contactos (Sluski, 1998) y el acceso a nuevos entornos (Gómez, 2012) que finalmente se irradiarían a la institución escolar, contribuyendo a la permanencia en ella.

Cuando se hace referencia a los vínculos en la escuela, se indica una relación que se establece entre los estudiantes, así como entre ellos y los adultos que hacen parte de la institución. Pero no es una relación cualquiera, sino parafraseando a Rogero (2012), una relación en la que se acompaña al estudiante para que alcance la autonomía, la madurez y la libertad. Desde esta perspectiva, se concibe que la inclusión requiere de cierto tipo de vinculación entre los actores de la escuela, un vínculo que debe tender a satisfacer las necesidades emocionales de primer orden, como lo son la afiliación, la pertenencia, la identidad y el apoyo (Montero y Fernández, 2011).

A partir de estos planteamientos, se podría definir el concepto de vínculos en la escuela como una forma de relación entre alumnos y entre alumnos-adultos, dando cuenta del carácter interdependiente de ambas partes, relación en la que emergen procesos de resiliencia, se mejora el rendimiento académico y se puede avanzar hacia la autonomía y la libertad necesaria para el desarrollo de todos y cada uno de los actores del contexto escolar (Alfageme, Cantos y Martínez, 2003).

En conclusión, la participación de los sujetos en los escenarios sociales, la posibilidad de pensarse como sujetos singulares, el desarrollo de sus competencias y el fortalecimiento de los víncu- 
los que establecen en los entornos de desarrollo, se constituyen en elementos característicos para plantear una revisión de la inclusión social en la escuela.

\section{Método}

Esta investigación se orienta a través de la técnica de investigación documental desde su dimensión cualitativa, en la cual se pretende reunir, sistematizar y analizar datos en forma de documentos producidos por la sociedad (Valles, 1997) para, en este caso, indagar sobre el fenómeno de la inclusión en la escuela.

\section{Fuentes documentales}

59 documentos: 46 artículos y 13 libros.

\section{Procedimiento}

El proceso de revisión inicia con la búsqueda en bases de datos desde las palabras clave: inclusión, exclusión, escuela, diversidad, multiculturalidad, integración; dada su relación con el fenómeno a estudiar.

Se identifican inicialmente 175 documentos, de ellos 157 artículos y 18 libros. La información obtenida se registra en una primera matriz en la que se identifica el año, mes, páginas, autor(es), título, revista, ISBN, número DOI, bibliografía relacionada con el fenómeno, resumen y ubicación del documento completo.

Posteriormente, se revisaron los documentos bajo los criterios acceso, actualidad (desde el 2005 a 2014) y relevancia en la comunidad científica en términos de indexación, de este proceso se obtienen 59 documentos.

Luego de definir las categorías singularidad, participación, vínculos y competencia, establecidas a partir de la revisión de las conceptualizaciones sobre inclusión, se desarrolla el proceso de análisis de contenido de tipo categorial, en una matriz orientada por los aspectos: tipo de documento, nombre, estrategia identificada, actores invo- lucrados, categorías deductivas (participación, competencia, vínculos y singularidad), categorías inductivas, anotaciones.

\section{Resultados}

\section{Competencias en la escuela}

En los contextos escolares hablar de competencia supone dos situaciones: reproducir una ideología y una forma de saber-hacer rígida e inflexible, o bien la formación de habilidades, actitudes, aptitudes, conocimientos y el reconocimiento e identificación de recursos, que posteriormente permitan la solución eficaz de problemas y la inclusión efectiva de niños y adolescentes a diferentes contextos.

De acuerdo con lo anterior, Roegiers (2000) sostiene que "la situación de aprendizaje, consiste nada menos que en dar al alumno la ocasión de ejercitar la competencia apuntada: un problema complejo para resolver, un trabajo de producción personal, una actividad de investigación, etc." (p. 115), frente a esto el mismo autor señala la importancia de que al formarse competencias se movilicen los recursos que se han integrado; que se propongan tareas significativas, es decir, que tengan un sentido; que las competencias se relacionen con una categoría de problemas específicos; que se generen situaciones nuevas, que, de acuerdo con Otálora (2011), representen un desafío en el que los estudiantes puedan ser creativos al buscar una solución.

Esto, en palabras de Otálora (2011), implica la construcción de un espacio educativo que resulte significativo para el sujeto, para lo cual es imprescindible la generación de situaciones que promuevan la construcción de nuevo conocimiento, caracterizado por el favorecimiento de formas de pensamiento más flexible y complejo que se relacione con los retos que afrontará el resto de su vida. Por lo tanto, esta autora propone la construcción de ambientes de aprendizaje significativos, caracterizados por: a) tener una estructura construida alrededor de unos objetivos y metas específicas; b) proporcionar una situación intensiva, vinculada con la resolución de problemas con metas de la cultura; c) construir un espacio en el 
que la situación sea extensiva, es decir, que facilite la manipulación compleja de metas; d) promover una situación con interacciones complejas; y e) desarrollar situaciones generativas, que involucren el despliegue de una serie de competencias, que le permitan al niño, niña o adolescente conocer y reconocer sus propios recursos, habilidades, aptitudes y conocimientos, para proponer soluciones a diferentes problemas que la vida presenta.

Lo anterior indica que un ambiente rico en experiencias, en situaciones que representen desafíos cada vez más complejos y que limite el ejercicio de trasmisión de saberes y el desarrollo de capacidades que se circunscriban a solo ciertos ejercicios, puede favorecer la preparación de niños y adolescentes capaces de comprender y transformar la realidad en la que viven (Perrenaud, 2006).

Con relación a la inclusión, se destaca la necesidad de involucrar las competencias como apuesta en la educación para favorecer este tipo de relación, ya que si bien su desarrollo se ha centrado en la dimensión individual estableciendo conexiones con el contexto como posibilitador, valdría la pena empezar a resaltar otras dimensiones:

El modelo más efectivo para el desarrollo de competencias es aquel en el cual los niños trabajan en colaboración, donde cada uno de ellos se transforma en un recurso al servicio de los demás y asume varios roles y responsabilidades en función de sus capacidades de comprensión y de su experiencia (Taylor et al., citado en Lansdown, 2005, p. 9).

De allí surge la comprensión de las competencias como comportamiento pro-social, lo cual involucra "la capacidad y voluntad de ayudar, compartir y cooperar, y participación social, es decir, la capacidad de responder a las propuestas sociales de los demás y de tomar iniciativas propias" (Rydell, Hagekull, y Bohlin, citado en Nyberg, Henricsson y Rydell, 2008, p. 643).

Es posible anclar a estas reflexiones estrategias como la concepción del niño, niña o adolescente como actor que participa de manera determinante en la construcción de relaciones de la sociedad desde su capacidad, competencia y creatividad
(Pilotti, 2000). Una apuesta por la persona y su responsabilidad en la toma de decisiones en cuestiones fundamentales que trascienden las aspiraciones del mercado, y que se logran a través del diálogo basado en el respeto y el afecto hacia los demás (Fielding, 2011). Y, la ayuda entre iguales que promueve el desarrollo afectivo y académico (CTROADI, 2008).

De otro lado, se evidencian estrategias referidas a la evaluación, en la cual se establece la importancia de evaluar en positivo la reflexión sobre las expectativas del profesorado hacia el alumnado, partiendo de la capacidad y la competencia y no de la deficiencia del alumno para que pueda conseguir el objetivo propuesto (CTROADI, 2008), tomar en consideración distintas variables que intervienen en el aprendizaje y el valor diferencial de cada una de ellas de acuerdo con la singularidad del estudiante (Echeita, Simón, Verdugo, Sandoval, López, Calvo y González, 2009), organización de actividades de refuerzo y planificación de las acciones de acceso al currículo general y adaptaciones curriculares individuales a partir de la estructuración de núcleos de competencias básicas, cognitivas, metodológicas y actitudinales (Peiró, 2008).

\section{Singularidad en la escuela}

La singularidad se convierte en el foco de atención de los procesos de inclusión social, ya que a través de esta se valida la identidad de cada uno y se favorece el bienestar (Wyn, 2009), por lo tanto, la comprensión de la escuela como un espacio de inclusión social permite el reconocimiento de las diferencias personales y las convierte en recursos para el aprendizaje, de manera que los estudiantes se sienten respetados, queridos y participes de los procesos que allí se gestan. Así, se contribuye en la construcción de una identidad personal y colectiva; personal en la medida en que el estudiante empieza a presentar y enriquecer una identidad y un proyecto de vida. Colectiva en el sentido de que se promueven valores como la equidad y la dignidad dentro de las escuelas (Peiró, 2008).

Como se puede apreciar, la inclusión social implica un cambio para las escuelas hacia procesos que 
validen, apoyen y utilicen la singularidad de los estudiantes como un recurso. Pero para hacerlo, es necesario que se asuma la tarea, como lo indica Echeita et al. (2009):

De identificar y remover, en su caso, las barreras que desde distintos planos de la vida escolar, al interactuar negativamente con las condiciones personales o sociales de los alumnos en determinados momentos, pudieran limitar en cada centro, precisamente, su presencia, aprendizaje o participación (p. 159).

Como parte de este cambio necesario y de la superación de barreras, es indispensable que se realice una modificación en la forma como los adultos comprenden a los niños y adolescentes. Para Lansdown (2005), la tarea de los adultos es incrementar y apoyar el desarrollo de las facultades y características personales, y para hacerlo se deben respetar las capacidades de los niños para definir sus propios intereses y prioridades, así como las estrategias para abordarlos. También es importante que se logre cooperación entre los niños y entre estos y los adultos. $Y$ por último, este autor resalta que el reconocimiento de la singularidad implica la creación de "oportunidades para que los niños formulen sus propias preocupaciones, prioridades e intereses" (p. 32).

Ahora bien, para dar respuesta a estas demandas que implican el reconocimiento de la singularidad, se requiere la creación de espacios y modelos basados en principios como la diferencia, la ciudadanía y la unidad en la diferencia (Peiró, 2008). A partir de estos principios es posible adoptar estrategias metodológicas que den respuesta a todos y cada uno de los estudiantes. Algunas de estas estrategias son: el aprendizaje cooperativo, la tutoría entre iguales, el aprendizaje por proyectos, el reconocimiento de los lenguajes verbales y no verbales, el contrato didáctico o pedagógico, los espacios para la realimentación y las prácticas flexibles (CTROADI, 2008). Además, es importante que se promueva el deporte y la recreación, ya que son una oportunidad para construir identidades propias e institucionales, para ampliar redes sociales, para enseñar el comportamiento ético y para reducir el aislamiento y el comportamiento antisocial (Cortis, 2009).
Para que los procesos de inclusión se favorezcan y se valide la singularidad de los estudiantes, es necesario que los maestros generen ciertos cambios al interior del aula. Estos cambios deben centrarse en generar un clima inclusivo donde todos se sientan acogidos y valorados; y el desarrollo de estrategias didácticas que garanticen el aprendizaje y participación de todos y cada uno de los estudiantes. Para que esto sea posible, Moliner (2008) propone que se deben "dar a conocer las singularidades individuales y en el caso en que no surja un apoyo entre iguales de forma espontánea, planificar la enseñanza de las competencias o habilidades necesarias para ello" (p. 40). Esto debido al tiempo y al tipo de interacción que tienen los maestros y los estudiantes.

Sin embargo, estas iniciativas no pueden limitarse a un maestro o aula en particular, sino que debe ser la escuela, en su conjunto, la que articule respuestas coherentes y globales a los retos que presenta la inclusión y que evalúe en qué medida los valores, los principios, las normas, las relaciones, las costumbres y las formas de participación que se gestan al interior de la escuela pueden llegar a facilitar u obstaculizar la inclusión social (González, 2008). De igual manera, esas acciones involucran a las familias, las comunidades y el Estado, los cuales deben empezar a generar iniciativas de inclusión a nivel local y general, en donde la comprensión de la singularidad sea un valor que identifique a toda la población y que se promueva en todos los ámbitos nacionales (Negron y Nett, 2005; Donelly y Coakley, 2002).

\section{Vínculos en la escuela}

Los vínculos concebidos como una forma de relación entre alumnos y entre estos y los adultos, implica que ellos no solo son concebidos como transmisores de conocimiento y ejemplo de comportamiento, sino que además se convierten en agentes en condiciones de potenciar la autogestión y autodirección de los niños, las niñas y los adolescentes (Alfageme, Cantos y Martínez, 2003).

Según esto, los adultos que interactúan con los niños en el contexto escolar deben empezar a asumir una postura diferente hacia ellos, ya que 
su intervención tiene efectos sobre el desarrollo y el aprendizaje que se construye en dichos espacios. Esta postura debe estar marcada por la confianza y debe proveer el tiempo y las habilidades necesarias para desarrollar relaciones con los estudiantes, al mismo tiempo que se permiten y se promueven las relaciones entre pares. Esto es aún más importante cuando se encuentran niños en situación de discapacidad, en donde se debe disponer más tiempo para que todos y cada uno de los actores escolares conozcan, comprendan y favorezcan la vinculación y participación de estos niños (Mitchell, Franklin, Greco y Bell, 2009).

Por lo tanto, el rol que adquieren los adultos en el contexto escolar es significativo para favorecer la madurez y autonomía de los estudiantes, de allí que Henderson y Milstein (2003, citado por Blanco, 2008) sugieran objetivos a trabajar con los estudiantes dirigidos a enriquecer los vínculos (tanto afectivos como de rendimiento): fijar límites claros y firmes, enseñar habilidades para la vida, construir resiliencia, brindar afecto y apoyo (estimular las relaciones de amistad entre pares y la relación entre escuela-comunidad), establecer expectativas elevadas y positivas y brindar oportunidades de participación significativas.

Pero esta labor no puede ser exclusiva del contexto escolar, ya que avanzar hacia la inclusión implica construir alianzas entre la escuela y la familia, en donde se realice "un compromiso compartido y recíproco en beneficio de un interés común: la educación de la infancia y la juventud" (Naradowski, 2008, p. 25). A partir de este planteamiento, la vinculación en la escuela debe ampliarse para incluir a todas las personas que de una u otra forma intervienen en el favorecimiento de la autonomía; de allí que sea necesario hacer partícipes a la familia y la comunidad en todo proceso de inclusión.

Ahora bien, para favorecer la vinculación en el contexto escolar, se requiere de varias estrategias, tanto dentro como fuera de la escuela. Una de ellas es el abordaje de la escuela desde un modelo intercultural, en donde se promueve la interacción con diferentes culturas y situaciones, de manera que se favorezca el sentido de pertenencia al mismo tiempo que se generan redes de apoyo; esto a partir del uso de estrategias de participación (Pei- ró, 2008). Así mismo, y dado el papel que juega la comunidad como actor significativo en el proceso de enseñanza-aprendizaje y en la autonomía de los niños, para Moliner (2008) es necesaria:

Una sensibilización a la opinión pública, analizando las políticas sociales y de empleo, teniendo en cuenta el papel de las asociaciones comunitarias y, por supuesto, reconociendo el importante papel de las familias en el apoyo y la difusión de la inclusión y sus implicaciones (pp. 29-30).

Como ya se ha visto, la vinculación es necesaria dentro de todo proceso de inclusión social, una vinculación que requiere de una relación entre los alumnos y entre estos y los adultos que intervienen en su desarrollo, de allí que sea importante incluir a las familias y a las comunidades. En este sentido existen varias estrategias que buscan promover esta vinculación alumno-escuela-familiacomunidad, algunas centradas en la participación de la familia, como lo son las escuelas de padres, talleres, proyectos, actividades complementarias, tertulias literarias, periódico escolar, fiestas (CTROADI, 2008); y otras enfocadas hacia la construcción de redes de colaboración, interdependencia, ayuda y apoyo mutuo con la comunidad que hace parte de la institución educativa o que interviene de alguna forma en ella (Echeita, 2008), tales como la celebración de convenios con entidades culturales, académicas y para la promoción de la salud, la participación en mesas de trabajo interinstitucionales y estatales para la construcción de políticas y la realización de eventos.

Por lo tanto, para favorecer los procesos de inclusión es necesario promover niveles de relación entre estudiantes y entre estos y los demás miembros de la escuela, a través de estrategias como la participación en equipos deportivos, culturales, académicos y extraescolares, que permitan reconocerse como sujetos multidimensionales, así como escenarios de expresión de sentimientos y expectativas en un ambiente de acogida y confianza. Para Roffey (2008, citado por Noaks y Noaks, 2009) el vínculo es un elemento clave para favorecer la relación alumno-alumno y alumno-maestro, ya que promueve la resiliencia y favorece los resultados académicos. De igual forma, se resalta la 
importancia de un tipo específico de vínculo; el que se construye a través de la mediación, ya que se convierte en una estrategia de inclusión social y en una técnica para la resolución de conflictos.

\section{Participación en la escuela}

La inclusión en la escuela hace referencia al concepto de participación, ya que el propósito de los procesos inclusivos la involucra como un logro sin ningún tipo de excepciones ni restricciones.

Para lograr la participación de la infancia es necesario que los diferentes actores del contexto escolar favorezcan espacios en los que puedan dar sus opiniones y tomar decisiones. Por medio de dichos espacios y en la medida en que mayor sea su participación, de acuerdo con Lansdown (2005), será posible enseñar a los niños la importancia de escucharse a sí mismos y a los demás, favoreciendo el valor de la democracia. De igual forma, adquieren experiencias que les permiten analizar las diferentes perspectivas y asumir responsabilidades por sus propias acciones.

La participación de la infancia cobra una importancia especial ya que las escuelas son contextos que van a favorecer u obstaculizar su desarrollo. Esto implica un cambio con respecto al rol que cumplen los niños en las escuelas, en el cual se exige un mayor compromiso en el reconocimiento de su papel activo en los diferentes procesos y espacios de la institución desde la diversidad que los caracteriza (Areiza, 2008). A partir de este cambio y de la comprensión de la diversidad y la infancia, es posible diseñar un nuevo sistema escolar, según Cuervo, Trujillo, Vargas, Mena y Pérez (2005):

En el que cada cual pudiera progresar, junto con otros, en función de sus necesidades particulares y que pueda adaptarse para satisfacer las necesidades educativas de cada alumno, de la mano de un profesorado que acepta y está capacitado para enfrentarse a la diversidad (p. 360).

En este sentido, la participación que debe tener la infancia en cualquier proceso inclusivo debe es- tar dirigida hacia la posibilidad de los estudiantes de estar informados sobre asuntos de la vida escolar que los afectan. Para lograrlo, es indispensable el apoyo de los adultos, en especial aquellos que hacen parte de la escuela, pues se encuentran en condiciones de otorgar espacios y oportunidades para que los niños influyan en los planes, los programas y los objetivos que se les proponen dentro y fuera del aula.

En este punto es conveniente resaltar los niveles de participación que propone Roger Hart (1997), citado por Alfageme, Cantos y Martínez (2003), en los que se busca un proceso progresivo hacia los más altos niveles de participación, entre los cuales se destaca el primer nivel de participación, en el cual los niños comprenden las intenciones del proyecto/actividad, saben quién tomó las decisiones sobre su participación y por qué, tienen un papel significativo, no decorativo; el segundo nivel de participación, en el que a veces los niños y jóvenes trabajan como consultores de los adultos de forma integral; el tercer nivel de participación, iniciado por los adultos, compartido con los niños y por lo tanto se produce un cambio importante pues los niños se involucran en el proceso completo; el cuarto nivel de participación, en el cual las actividades son iniciadas y dirigidas por los niños sin intervención de los adultos; y un último nivel, en el que las actividades son iniciadas y dirigidas por los niños y las decisiones son compartidas con los adultos, quienes les apoyan para llevarlas a cabo sugiriendo formas de mejorarla.

A partir de estos grados es posible identificar diferentes estrategias que favorezcan los procesos de participación, no solo de la infancia, sino de todos los actores del contexto escolar, tales como "los alumnos ayudantes" y el trabajo por proyectos. La primera se enfoca hacia la creación de un grupo dentro del aula de varios estudiantes a quienes se capacite en el diálogo y la resolución de conflictos y se les otorgue la tarea de favorecer la inclusión de todos sus compañeros, intervenir en cualquier dificultad entre iguales y contribuir en la construcción y promoción de normas de convivencia. La otra estrategia, el desarrollo de temáticas por proyectos, busca plantear el conocimiento de la realidad de modo globalizado e interdisciplinar. 
Consiste en generar espacios de trabajo en los que los estudiantes aprendan procedimientos que les ayuden a organizar, comprender y asimilar una información y es el profesor quien orienta las dudas que surgen en el proceso (CTROADI, 2008).

Similar a la estrategia del alumno ayudante se encuentra la creación de "Consejos de los Niños", integrados por niños de distintas instituciones escolares de la localidad. El propósito de este consejo es que los niños se incluyan en las discusiones de los problemas que afectan a sus escuelas (Corvera, 2011).

Por otro lado, en las estrategias dentro del aula, Conole (2012) propone el uso de los nuevos medios sociales y participativos que ha aportado la tecnología y que proporcionan nuevas formas de comunicación y colaboración. Además, para este autor es necesario utilizar metodologías pedagógicas enfocadas en el aprendizaje constructivista y situado, como lo son el aprendizaje basado en investigaciones y el aprendizaje dialógico y reflexivo, ya que proveen mayor libertad y se enfocan en los niños y adolescentes como sujetos competentes en los procesos de enseñanza-aprendizaje.

Por último, se encuentra la creación de espacios fuera del aula, pero que influyen en los procesos y contenidos que se trabajan en las diferentes asignaturas. Uno de estos espacios es el de refuerzo, coordinado por el maestro y cuyo objetivo es ayudar a superar las dificultades de aprendizaje que se detecten individual o grupalmente en cada evaluación y que en su desarrollo pueden concretarse en una diversidad de medidas que van desde el uso de actividades grupales como el aprendizaje cooperativo, la creación de grupos de trabajo en horarios extra clase, hasta las acciones dirigidas por el docente como el trabajo personal tutorizado y la intervención de dos profesores en una misma aula. El otro espacio es la optatividad, orientado a la diversidad y singularidad en la que se ofrecen materias alternas entre las que los estudiantes pueden elegir y que se definen según las necesidades e intereses del alumnado, los conocimientos del profesorado y los recursos disponibles (Martínez, 2005).

\section{Conclusiones}

Las estrategias identificadas en los documentos revisados a la luz de las cuatro categorías establecidas en la investigación, se destacan por su conexión en una lógica de la inclusión que va más allá de la comprensión de la diversidad desde el paradigma de la "alteridad", en el cual el foco se halla en los grupos de personas que son leídos como diferentes frente a una cultura hegemónica (Batallán y Campanini, 2007).

De allí que si bien muchas de las estrategias son extraídas de documentos relacionados con temas como diversidad y discapacidad, es importante destacar el avance en las investigaciones y construcciones sobre la inclusión al poner el acento en el reconocimiento de la singularidad que emerge en la escuela para generar tensiones que invitan al cambio, lo que por supuesto, se hace más visible en grupos determinados de estudiantes considerados como el "otro" diferente debido a sus capacidades contrastantes con aquellas que son usuales (Figueroa, 2011), orientación sexual, cultura, etc., pero que a su vez, generan los escenarios para repensar los procesos de inclusión en la escuela anclada a su democratización desde el respeto a la diversidad de manera recíproca (Batallán y Campanini, 2007), es decir, no desde el foco sobre las minorías que están al margen de la cultura dominante, sino del reconocimiento de los actores de la escuela como seres únicos miembros de una sociedad.

De esta manera, las estrategias identificadas en la categoría singularidad, llevan a la necesidad de su expresión a través de escenarios respetuosos y enriquecedores, lo que a su vez favorece el desarrollo de una identidad personal y colectiva que conlleva al logro de diferentes aprendizajes. Por lo tanto, es posible hallar la relación entre la singularidad y las competencias, en tanto que las estrategias referidas a esta categoría se anclan en la importancia de generar retos y situaciones problema que inviten al estudiante a usar y transferir el conocimiento propio del contexto escolar a otros contextos de su vida cotidiana y viceversa. En ese sentido, el reconocimiento de la singularidad potenciaría las situaciones problema 
presentadas en la escuela, en tanto se anclan a la vida de los niños y adolescentes.

De otro lado, se destaca en esta categoría un avance teórico sobre la conceptualización de las competencias y su gestión en la escuela, dado que en las estrategias identificadas se incluyen aspectos como la cooperación entre pares, el diálogo y el afecto, pues ser competente para el contexto implica necesariamente establecer relaciones con características que le indiquen al estudiante que pertenece allí. Aspecto que involucra la categoría vínculos, dado que el sentido de pertenencia, escenarios marcados por la confianza en los que se expresen necesidades, intereses y expectativas y la promoción del afecto parecen hacen parte de contextos para la formación de competencias que invitan a la evaluación en positivo y al reconocimiento de los recursos de cada actor de la escuela.

En cuanto a la categoría participación, que es una de las más evidentes en los desarrollos teóricos sobre la inclusión, se destacan estrategias que al parecer se anclan a la singularidad, los vínculos y las competencias, dado que para ser partícipe en un contexto, se deben generar escenarios de confianza en los cuales se exprese la identidad, se generen conexiones emocionales y se permita exponer las capacidades, habilidades, conocimientos e intereses.

Finalmente, en las estrategias se destaca el papel de los profesores en la gestión de la inclusión, dado que son ellos a quienes se atribuye el papel de la educación en la escuela. Lo cual presenta una tensión sobre la que se debe ahondar, ya que muchas de las tareas que se destacan en los textos deben ser agenciadas por ellos, en un marco institucional que se sobreentiende como preparado para la inclusión, pero que no necesariamente es así. De ahí la importancia de profundizar en la formación docente, la corresponsabilidad de las instituciones, la investigación pedagógica y las políticas.

Por lo tanto, se considera que la inclusión social en contextos educativos exige cambios estructurales profundos, ya que las estrategias presentadas requieren unos soportes que van desde la concepción de la evaluación, que en la actualidad necesariamente está anclada a demandas estan- darizadas y que podría obstaculizar los avances en la promoción de la singularidad y las competencias, pasando por los significados sobre la infancia y la adolescencia que desde las posturas evolutivas tradicionales despojan de los atributos para la participación a los niños y adolescentes, hasta la estructura social y económica que establece formas de relación entre padres e hijos, profesores y estudiantes, padres y profesores condicionadas por el valor económico del tiempo y las urgencias del capital.

\section{Transferencia a la práctica}

Las iniciativas que se han generado en torno a la inclusión en la escuela pueden agruparse en dos categorías: una centrada en la importancia de los niños y adolescentes, y otra enfocada a favorecer la necesaria interacción entre escuela y familia, por lo tanto, la inclusión social en contextos educativos debe orientarse hacia el reconocimiento, promoción y participación de la multiplicidad, singularidad y diversidad de actores, identidades, culturas y contextos propios del territorio, ya que es precisamente esta diversidad la que enriquece las experiencias, y por ende, los aprendizajes que se pueden construir en la escuela.

\section{Referencias}

Alfageme, E., Cantos, R., y Martínez, M. (2003). De la participación al protagonismo infantil: propuestas para la acción. Madrid: Plataforma de Organizaciones de Infancia.

Americas Quarterly. (2013). Índice de inclusión social. 7(3), 3-15.

Areiza, Y. (2008). La inclusión escolar: ¿un compromiso de quién? [Versión electrónica]. Poiésis, (16), 1-5. Recuperado de http://www. funlam.edu.co/poiesis/Edicion016/Inclusionescolar.YulianaAreiza.pdf

Batallán, G., y Campanini, S. (2007). El "respeto a la diversidad" en la escuela: atolladeros del relativismo cultural como principio moral. Revista de Antropología Social, (16), 159-174. 
Bernal, T., Figueroa, X., Gutiérrez de Piñeres, C., y Jaramillo, J. (2012). Análisis de estrategias participativas para promover la movilización de significados y acciones en torno a la inclusión social de niños y adolescentes en contextos familiares, escolares y de protección estatal. Universidad Santo Tomás: Proyecto de investigación presentado al FODEIN. Documento no publicado.

Blanco, M. (2008). Construyendo las bases de inclusión y la calidad de la educación en la primera infancia [versión electrónica]. Revista de Educación, (347), 33-54. Recuperado de http://www.revistaeducacion.mec.es/re347/ re347_02.pdf

Blanco, V. (2008). Derechos de la infancia (0-6 años) y educación inclusiva [versión electrónica]. Revista de Educación, (347), 55-75. Recuperado de http://www.revistaeducacion. mec.es/re347/re347_03.pdf

Blanco, R. (2006). La equidad y la inclusión social: uno de los desafíos de la educación y la escuela hoy. REICE: Revista Electrónica Iberoamericana sobre Calidad, Eficacia y Cambio en Educación, 4(3), 1-15, Recuperado de http:// www.rinace.net/arts/vol4num3/art1.pdf

Booth, T., Ainscow, M., Black-Hawkins, K., Vaughan, M., \& Snow, L. (2000). Index for inclusión: Developing learning and participation in schools. Bristol, Center for Studies on Inclusive Education.

Centro Territorial de Recursos para la Orientación, la Atención a la Diversidad y la Interculturalidad - CTROADI. (2008). Inclusión social: estrategias organizativas y metodológicas. Albacete, España. Recuperado de http://www.laroda.es/ discapacidad/imagenes/INCLUSI\%C3\%93N\%20 SOCIAL.pdf

Conole, G. (2012). Fostering social inclusion through open educational resources (OER) [versión electrónica]. Distance Education, 33(2), 131134. DOI: $10.1080 / 01587919.2012 .700563$.

Corcuff, P. (2009). Pierre Bourdieu (1930-2002) leído de otra manera. Crítica Social Post -
Marxista y el problema de la singularidad individual. Revista Cultura y Representaciones Sociales. 4(7), 9-26. Recuperado de http:// revistas.unam.mx/index.php/crs/article/ view/16401/15610

Cortis, N. (2009). Social inclusion and sport: Culturally diverse women's perspectives [versión electrónica]. The Australian Journal of Social Issues, 44(1), 91-106. Recuperado de http:// search.informit.com.au/documentSummary;d $\mathrm{n}=865644299270825$;res=IELFSC

Corvera, N. (2011). Participación ciudadana de los niños como sujetos de derechos [versión electrónica]. Persona y sociedad, 25(2), 73-99. Recuperado de http://Www.Personaysociedad. $\mathrm{Cl} /$ Participacion-Ciudadana-De-Los-Ninos-Como-Sujetos-De-Derechos/

Cuervo, C., Trujillo, A., Vargas, D., Mena, B., y Pérez, L. (2005). Discapacidad e inclusión social, reflexiones desde la Universidad Nacional de Colombia. Bogotá: Universidad Nacional.

Chana, L. (2007). Promoviendo vínculos... tejiendo redes: claves para la inclusión social y educativa de la infancia en dificultades. Trabajo social hoy, 50, 99-117.

Donelly, P., \& Coakley, J. (2002). The role of recreation in promoting social inclusion. Toronto, Canadá: The Laidlaw Foundation.

Echeita, G. (2008). Inclusión y exclusión educativa: voz y quebranto. REICE: Revista Electrónica Iberoamericana sobre Calidad, Eficacia y Cambio en Educación, 6(2), 9-18. Recuperado de http://www.redalyc.org/articulo.oa?id $=55160202$

Echeita, G., Simón, C., Verdugo, M., Sandoval, M., López, M., Calvo, I., y González, F. (2009). Paradojas y dilemas en el proceso de inclusión educativa en España. Revista de Educación, (349), 137-152.

Fielding, M. (2011). La voz del alumnado y la inclusión educativa: una aproximación democrática radical para el aprendizaje intergeneracio- 
nal. Revista interuniversitaria de formación del profesorado, 70, 31-62.

Figueroa, M. (2010). Significados acerca de la atención educativa en el aula regular a niños y niñas en situación de discapacidad en tres instituciones educativas. Diversitas: perspectivas en psicología, 7(2), 321-333.

Figueroa, M. (2012). Diversidad e inclusión escolar: retos para la psicología educativa actual. En G. Marciales (comp). Psicología Educativa: trayectorias, convergencias y vórtices. Colección Saber, Sujeto y Sociedad. Editorial Pontifica Universidad Javeriana.

Gairín, J., e Iglesias, E. (2008). La interacción docente-discente en contextos escolares con fuerte presencia de alumnos de familia inmigrante: propuestas de la investigación. Revista Iberoamericana de Educación, (48), 233-252.

Gómez, A. (2012). Familias de acogida y niños y adolescentes en protección: vínculos y tutores de resiliencia. En Libro de ponencias: $V$ Congreso mundial de infancia y adolescencia. San Juan, Argentina.

González, M. (2008). Diversidad e inclusión educativa: algunas reflexiones sobre el liderazgo en el centro escolar. REICE: Revista Electrónica Iberoamericana sobre Calidad, Eficacia y Cambio en Educación, 6(2), 82-99. Recuperado de http://www.redalyc.org/articulo. oa?id $=55160208$

Jansen, W., Otten, S., Van Der Zee, K., \& Lise, J. (2014). Inclusion: Conceptualization and measurement. European Journal of Social Psychology, 44(4), 370-385.

Lansdown, G. (2005). ¿Me haces caso? El derecho de los niños y las niñas a participar en las decisiones que los afectan, Cuadernos sobre Desarrollo Infantil Temprano, (36). La Haya, Países Bajos: Fundación Bernard van Leer.

Martín, E., y Solari, M. (2011). ¿Puede el enfoque de las competencias contribuir a la inclusión y a la ciudadanía? Tejuelo, 12, 9-25.
Martínez, B. (2005). Las medidas de respuesta a la diversidad: posibilidades y límites para la inclusión escolar y social. Revista de curriculum y formación del profesorado, 9(1), 1-31.

Mitchell, W., Franklin, A., Greco, V., \& Bell, M. (2009). Working with children with learning disabilities or who communicate non-verbally: Research experiences and their implications for social work education, increased participation and social inclusion [versión electrónica]. Social Work Education, 28(3), 309-324. DOI: $10.1080 / 02615470802659530$

Moliner, M. (2008). Condiciones, procesos y circunstancias que permiten avanzar hacia la inclusión educativa. REICE: Revista Electrónica Iberoamericana sobre Calidad, Eficacia y Cambio en Educación, 6(2), 27-44. Recuperado de http:// www.rinace.net/arts/vol6num2/art3.pdf

Montero, D., y Fernández, P. (2011). Calidad de vida, inclusión social y procesos de intervención. Bilbao, España: Publicaciones de la Universidad de Deusto.

Moriña, A., y Parrilla, A. (2006). Criterios para la formación permanente del profesorado en el marco de la educación inclusiva. Revista de Educación, (339), 517-539.

Naradowski, M. (2008). La inclusión educativa. reflexiones y propuestas entre las reflexiones, las demandas y los slogans. REICE: Revista Electrónica Iberoamericana sobre Calidad, Eficacia y Cambio en Educación, 6(2), 19-26. Recuperado de http://www.rinace.net/arts/ vol6num2/art2.pdf

Negron, B., y Nett, M. (2005). Diversidad cultural: el valor de la diferencia. Santiago de Chile: LOM Ediciones.

Nyberg, L., Henricsson, L., \& Rydell, A. (2008). Low social inclusion in childhood: Adjustment and early predictors. Infant and Child Development, 17(6), 639-656.

Noaks, J., y Noaks, L. (2009). School-based peer mediation as a strategy for social inclusion 
[versión electrónica]. Pastoral Care in Education, 27(1), 53-61. DOI: 10.1080/02643940 902731880

Oakley, P. (1995). Los orígenes europeos de la exclusión social. Aplicación a los países en desarrollo. En Buvinic, M, Mazza, J., y Pungiluppi, J. (2004). Inclusión social y desarrollo económico en América Latina. Colombia: IDB.

OCDE. (2005). La definición y selección de competencias clave (DeSeCo). Resumen. Recuperado de http://www.deseco.admin.ch/bfs/deseco/ en/index/02.html

Oraisón, M., y Pérez, A. (2006). Escuela y participación: el difícil camino de la construcción de ciudadanía. Revista Iberoamericana de Educación. Organización de Estados Iberoamericanos para la Educación, la Ciencia y la Cultura (OEI), (42), 15-29.

Otálora, J. (2011). Diseño de espacios significativos para el desarrollo de competencias en la infancia. CS, 5, 71-96.

Peiró, S. (2008). Multiculturalidad escolar y convivencia educativa. Alicante, España: Editorial Club Universitario.

Perrenaud, P. (2006). Construir competencias desde la escuela. Chile: Dolmen.

Pilotti, F. (2000). Globalización y convención sobre los derechos del niño. OEA.

Roegiers, X. (2000). Saberes, capacidades y competencias en la escuela: una búsqueda de sentido. Innovación educativa, (10), 103-119.
Rogero, J. (2012). Las limitaciones del proceso de inclusión. En La educación formal y los procesos de inclusión social, (pp. 35-62). Madrid: Caritas Española.

Sen, A. (2000). Desarrollo y libertad. Barcelona: Planeta.

Sluski, C. (1998). La red social: de la frontera a la práctica sistémica. Barcelona: Gedisa.

Shore, L., Randel, A., Chung, B., Dean, M., Holcombe, K., \& Singh, G. (2011). Inclusion and diversity in work groups: A review and model for future research. Journal of Management, 37(4), 1262-1289.

UNESCO. (2005). Políticas educativas de atención a la diversidad cultural. Organización de las Naciones Unidas para la Educación, la Ciencia y la Cultura. Santiago, Chile: UNESCO.

Valles, M. (1997). Técnicas cualitativas de investigación social. Reflexión metodológica y práctica profesional. Madrid: Síntesis.

Varela, J., y Ribes, E. (2002). Aprendizaje, inteligencia y educación. En E. Ribes Iñesta (Ed.). Psicología del aprendizaje. México: Manual Moderno.

Wyn, J. (2009). The changing context of Australian youth and its implications for social inclusion. Youth Studies Australia, 28(1), 46-50. 